Their study included 154 patients with SLE, 48 patients with RA, and 72 healthy controls. The HDL of SLE patients was more proinflammatory than the HDL of RA patients, which in turn was more proinflammatory than the HDL of controls (mean 1.02 FU, 0.81 FU, and 0.68 FU on lipidoxidation fluorescence assay, respectively; all $P \leq 0.016)$. A similar pattern was observed in the numbers of patients with proinflammatory HDL 21.0FU: more SLE patients than RA patients, and more RA patients than controls, had proinflammatory HDL above this threshold (44.7\%, $20.1 \%$ and $4.1 \%$, respectively; all $P<0.006$ ). In all groups, levels of oxidized LDL correlated positively with levels of HDL.

Fourteen patients, all with SLE, had a history of atherosclerosis. The mean level of proinflammatory HDL in these patients was significantly higher than in patients without a history of atherosclerosis $(P=0.01)$.

McMahon et al. suggest that treating SLE and RA patients who have high levels of proinflammatory HDL and oxidized LDL with therapies that restore the protective function of HDL might help prevent the development of atherosclerosis.

Original article McMahon M et al. (2006) Proinflammatory high-density lipoprotein as a biomarker for atherosclerosis in patients with systemic lupus erythematosus and rheumatoid arthritis. Arthritis Rheum 54: 2541-2549

\section{Risk factors for vertebral fracture}

The prevalence of vertebral fracture in the white US population $\geq 80$ years old has been estimated at between $45 \%$ and $53 \%$ in women, and between $40 \%$ and $50 \%$ in men. Despite this high prevalence, the etiology of vertebral factures remains unclear. Samelson et al. have conducted the first prospective study of potential risk factors at middle age, and assessed their predictive power for vertebral fracture later in life.

The study included 452 women and 252 men for whom serial radiographs of the lumbar and thoracic spine at baseline and at a follow-up visit were available. Overall, $24 \%$ of women and $10 \%$ of men had at least one vertebral fracture during the 25-year study period. Although having suffered a previous fracture was not a risk factor for subsequent fracture, a previous fracture of at least moderate severity increased the risk of a subsequent moderate-to-severe fractureby five times in women and by six times in men (odds ratios 5.00, 95\% Cl 1.05-23.78 and 6.65, $95 \% \mathrm{Cl} 1.47-30.03$, respectively). Alcohol consumption increased fracture risk approximately fourfold in men $(P=0.0099)$, and there was a trend for smoking and alcohol consumption to increase fracture risk in women.

It is unclear why no other lifestyle factors had an impact on vertebral fracture; however, the authors suggest that their results might have been subject to a survival bias, or affected by the small sample size.

Original article Samelson EJ et al. (2006) Incidence and risk factors for vertebral fracture in women and men: 25-year follow-up results from the population-based Framingham study. J Bone Miner Res 21: 1207-1214

\section{Which biomarkers predict progression of knee OA?}

A single measurement of serum hyaluronicacid level, or short-term monitoring of urinary C-telopeptide of type II collagen (CTX-II) levels, can predict which patients with knee osteoarthritis $(\mathrm{OA})$ are most likely to suffer disease progression, say Bruyere and colleagues.

This prospective study enrolled 62 patients ( 49 female) with medial-compartment knee OA. The team assessed the ability of levels of biomarkers of bone, cartilage, and synovium (namely serum hyaluronic acid, osteocalcin, cartilage glycoprotein 39 , cartilage oligomeric matrix protein, and urinary CTX-I and CTX-II)—obtained at baseline and after 3 months - to predict progression of knee OA over 1 year. At baseline and follow-up, the volume and thickness of the cartilage of the femur, medial tibia, and lateral tibia was assessed with MRI, and a whole-organ MRI score of the knee OA was obtained.

Changes in cartilage volume and thickness after 1 year were not associated with baseline levels of any of these markers; however, an increased level of CTX-II at 3 months was markedly associated with a decreased mean thickness of medial and lateral tibial cartilage. A worsened whole-organ MRI score at 1-year follow-up was predicted by elevated levels of hyaluronic acid at baseline. As hyaluronicacid levels are a measure of synovial inflammation, the authors highlight the potential importance of the synovium in the disease course of $\mathrm{OA}$, and emphasize the potential 